\title{
EL PODER DEL AUTOPERDÓN EN LA PANDEMIA COVID-19: FUTURA AGENDA DE INVESTIGACIÓN
}

\author{
Celeste León-Moreno \\ Universidad Pablo de Olavide (UPO), Sevilla \\ cmleomor@upo.es \\ Ana Romero-Abrio \\ Universidad Internacional de la Rioja (UNIR) \\ ana.romero@unir.net \\ Belén Martínez-Ferrer \\ Universidad Pablo de Olavide (UPO), Sevilla \\ bmarfer2@upo.es
}

Recepción Artículo: 17 mayo 2021

Admisión Evaluación: 17 mayo 2021

Informe Evaluador 1: 26 mayo 2021

Informe Evaluador 2: 28 mayo 2021

Aprobación Publicación: 02 junio 2021

\section{RESUMEN}

En este trabajo se presentan algunas líneas de investigación futuras para examinar los beneficios del autoperdón como una estrategia de afrontamiento proactiva que puede ayudar a las personas a valorar de una forma más objetiva su percepción de culpabilidad o de responsabilidad sobre un daño (real o imaginario) hacia los demás 0 hacia sí mismas durante la actual pandemia del COVID-19.

Palabras clave: COVID-19; autoperdón; autoaceptación; futuras líneas de investigación

\section{ABSTRACT}

The power of self-forgiveness in the covid-19 pandemic: future research agenda. This paper presents new lines of future research to examine the benefits of self-forgiveness as a proactive coping strategy to help people assess more objectively any feelings of guilt or responsibility for harm (real or imaginary) towards others or towards themselves during the current COVID-19 pandemic.

Keywords: COVID-19; self-forgiveness; self-acceptance; new lines of future research

\section{INTRODUCCIÓN}

La actual pandemia del COVID-19 genera ansiedad, estrés y malestar en la población debido a los sentimientos de incertidumbre y de confusión, así como a los riesgos de contagio y muerte (Duan y Zhu, 2020). En este contexto, algunas personas pueden experimentar sentimientos negativos como impotencia, rabia o sentimientos de culpabilidad por la percepción de responsabilidad y arrepentimiento sobre un daño causado (real o imagina- 


\section{EL PODER DEL AUTOPERDÓN EN LA PANDEMIA COVID-19: FUTURA AGENDA DE INVESTIGACIÓN}

rio) a otras personas o hacia sí mismas. Según Entight (2015) en situaciones graves como, por ejemplo, el duelo por la muerte de un ser querido, las personas pueden sentirse culpables e incapaces de superar la situación. ¿Cómo puede la búsqueda del autoperdón y de la autoaceptación ayudar a afrontar las graves consecuencias de la pandemia del COVID-19 en la salud y el ajuste psicosocial de la población? En este trabajo proponemos algunas líneas de investigación futuras para explorar el proceso de afrontamiento de la pandemia desde el autoperdón y la autoaceptación.

\section{¿QUÉ ES EL AUTOPERDÓN?}

El perdón a uno mismo alude al deseo de abandonar el autoresentimiento ante la percepción de haber cometido una transgresión , fomentando la compasión, la generosidad o el amor hacia sí mismo (Enright y The Human Development Study Group, 1996). Éste puede ser entiendo como una conducta específica, dirigida a un daño concreto, en el que la persona ha dañado a otros o a sí misma (perdón específico), o como un rasgo de personalidad, una tendencia a perdonarse en distintas situaciones y a lo largo del tiempo (perdón disposicional) (Prieto-Ursúa y Echegoyen , 2015). Sin embargo, el autoperdón no es lo mismo que ser autoindulgente , minimizar o excusar los fallos y daños causados (Pallarés, 2006).

\section{¿POR QUÉ ES NECESARIO EXPLORAR LOS BENEFICIOS DEL AUTOPERDÓN EN LA PANDEMIA DEL COVID-19?}

Según Woodyatt y Wenzel (2017) el autoperdón es relevante cuando una persona percibe que ha dañado a otras personas 0 a sí misma en contra de sus propios valores, e incide en tres de sus necesidades psicológicas:

En primer lugar, el sentido de pertenencia y de apoyo de otras personas (Baumeister y Leary, 1995). Como expone Extremera (2020), las condiciones de aislamiento social y confinamiento obligatorio han podido poner a prueba esos vínculos y lazos afectivos interpersonales. En la medida en que esta necesidad no se satisface, puede derivar en sentimientos de culpabilidad y autocondena, por ejemplo: sentimientos de culpabilidad por no poder visitar a algún miembro de la familia; sentimientos de culpabilidad por una ruptura durante la pandemia, entre otros. En ese sentido, en futuros estudios sería interesante explorar si las condiciones de aislamiento social y confinamiento obligatorio han mermado el sentido de pertenencia y de apoyo de algunas personas, y si el autoperdón ha podido contribuir a fomentar sentimientos positivos hacia sí mismas (por ejemplo: la autocompasión, la autoeficacia y el autocontrol).

En segundo lugar, la necesidad de agencia que está relacionada con el deseo de sentir que tenemos el control de nosotros mismos, de nuestras acciones y de nuestras vidas. Es probable, que algunas personas perciban que no han cumplido adecuadamente con las medidas de protección de contagio (por ejemplo, uso correcto de la mascarilla, lavarse las manos con regularidad, mantener la distancia de seguridad, etcétera). Según Pallarés (2016) algunas personas necesitan perdonarse a sí mismas incluso cuando no ha habido un daño real o intencionado, pero experimentan sentimientos de culpabilidad con mayor o menor intensidad. Creemos que otra línea interesante de investigación seria examinar si a pesar de que en algunos casos los sentimientos negativos hacia sí mismos pueden ser irracionales, el autoperdón puede ser necesario y positivo para las personas que experimentan un sentimiento de culpa irracional o es más recomendable ayudarle a comprender su ausencia de culpa y responsabilidad en línea con el planteamiento de Enright (2015).

En tercer lugar, la necesidad de tener una vida con sentido (Graham, Morse, O'Donnell y Steger, 2017). Graham et al. (2017) describen el sentido de la vida como los juicios subjetivos de las personas (a) de que sus vidas tienen sentido y son valiosas, (b) de que tienen sueños, metas y aspiraciones a conseguir (es decir, propósito), y (c) de que sus vidas tienen un valor innato y vale la pena vivirlas. Es probable que la pandemia para algunas personas sea una oportunidad de crecimiento personal y autorreflexión sobre el sentido de sus vidas. En futuros trabajos seria muy interesante corroborar si durante la pandemia las personas se han cuestionado sobre el propósito de sus vidas y el rol que tiene el autoperdón en este proceso. 


\section{¿CÓMO PERDONARSE A SÍ MISMO?}

\section{a) Cómo pasar de la rumiación a la valoración objetiva de responsabilidad:}

Como hemos señalado previamente algunas personas pueden experimentar continuos sentimientos de arrepentimiento, resentimiento, vergüenza y culpa por incidentes relacionados con la pandemia. En este sentido, el autoperdón efectivo requiere que la persona se responsabilice por su transgresión (Woodyatt, Wenzel y de Vel Palumbo, 2017). Sin embargo, es probable que en muchos casos puedan tener un sentido de responsabilidad inapropiado o desproporcionado porque determinadas situaciones están fuera de su control (Woodyatt, Wenzel, y Griffin, 2017). Pero, ¿qué ocurre cuando se hacen esas atribuciones de una forma irracional? ¿Sería necesario disculparse públicamente? De acuerdo con Enright (2015), en estos casos, el autoperdón puede implicar el reconocimiento de los propios límites personales por los acontecimientos negativos y un proceso de liberación del resentimiento hacia uno mismo por una mala acción percibida. No obstante, hay situaciones en las que la responsabilidad personal es muy ambigua, este paso puede resultar muy difícil y doloroso. En consecuencia, en futuros trabajos creemos que es interesante profundizar en las implicaciones que tiene la inteligencia emocional en la percepción de responsabilidad y sus relaciones con la búsqueda del autoperdón y de la autoaceptación.

\section{b) Pasar del remordimiento a la restauración:}

Cuando las personas exploran y aceptan la responsabilidad por el daño causado a otros o hacia si mismos suele ir acompañado de sentimientos de remordimiento, culpa, o arrepentimiento por su ofensa (Cornish y Wade, 2015a). Aunque estas emociones pueden derivar en el deseo de reparar o restituir los hechos (Fisher y Exline, 2006), algunas personas necesitan ayuda para reducir sus sentimientos de autocrítica y para poder centrarse en los sentimientos de arrepentimiento por la ofensa (Cornish y Wade, 2015b). El remordimiento por las acciones de las que uno es responsable puede utilizarse como motivación para participar en el tercer componente del autoperdón, que es la restauración (Cornish y Wade, 2015b). La restauración implica los intentos de reparar el daño causado por el evento hiriente y un nuevo compromiso con los valores propios que pueden haber sido violados por la ofensa (Woodyatt y Wenzel, 2014). De hecho, Hall y Fincham (2005) señalan que, sin admitir implícita 0 explícitamente que nuestra conducta ha sido incorrecta y sin admitir la responsabilidad o la culpa por dicha conducta, es imposible que tenga lugar un auténtico perdón a uno mismo. En este sentido resultaría de gran interés explorar cómo se deben comunicar las disculpas para que sean efectivas, y los motivos subyacentes que existen a la resistencia a disculparse y a pedir perdón.

\section{REFERENCIAS BIBLIOGRÁFICAS}

Baumeister, R. F. y Leary, M. R. (1995). The need to belong: desire for interpersonal attachments as a fundamental human motivation. Psychological bulletin, 117(3), 497.

Blustein, J. (2000). On taking responsibility for one's past. Journal of Applied Philosophy, 17, 1-19.

Cornish, M. A. y Wade, N. G. (2015a). A therapeutic model of self-forgiveness with interventionstrategies for counselors. Journal of Counseling and Development, 93, 96-104.

Cornish, M. A. y Wade, N. G. (2015b). Working through past wrongdoing: Examination of a self-forgiveness counseling intervention. Journal of Counseling Psychology, 62, 521-528.

Duan, L. y Zhu, G. (2020). Psychological interventions for people affected by the COVID-19 epidemic. The Lancet Psychiatry, 7(4), 300-302.

Enright, R. (2015). Las 8 claves del perdón. Barcelona: Editorial Eleftheria

Enright, R. D. y The Human Development Study Group. (1996). Counselling within the forgive-ness triad: On forgiving, receiving forgiveness, and self-forgiveness. Journal of Counselling and Values, 40, 107-126.

Extremera, N. (2020). Afrontando el estrés causado por la pandemia del COVID-19: Futura agenda de investigación desde la inteligencia emocional. International Journal of Social Psychology, 35(3), 631-638. 


\section{EL PODER DEL AUTOPERDÓN EN LA PANDEMIA COVID-19: FUTURA AGENDA DE INVESTIGACIÓN}

Fisher, M. L. y Exline, J. J. (2006). Self-forgiveness versus excusing: The roles of remorse, effort, and acceptance of responsibility. Self and Identity, 5(2), 127-146.

Gerber, L. A. (1990). Transformations in self-understanding in surgeons whose treatment efforts were not successful. American Journal of Psychotherapy, 44, 75-84.

Graham, K. L., Morse, J. L., O'Donnell, M. B. y Steger, M. F. (2017). Repairing meaning, resolving rumination, and moving toward self-forgiveness. In Handbook of the psychology of self-forgiveness (pp. 59-72). Springer, Cham.

Hall, J. H., y Fincham, F. D. (2005). Self-forgiveness: The stepchild of forgiveness research. Journal of Social and Clinical Psychology, 24, 621-637.

Luskin, F. (2002). Forgive for good. New York, NY: HarperCollins.

Nelissen, R. y Zeelenberg, M. (2009). When guilt evokes self-punishment: Evidence for the existence of a dobby effect. Emotion, 9(1), 118-122.

Pallarés, E. (2016). El perdón como fortaleza humana. Bilbao: Editorial Mensajero

Prieto-Ursúa, M. y Echegoyen, I. (2015). ¿Perdón a uno mismo, autoaceptación o restauración intrapersonal? Cuestiones abiertas en Psicología del perdón. Papeles del psicólogo, 36(3), 230-237.

Tangney, J., Boone, A. L. y Dearing, R. (2005). Forgiveness of the self: Conceptual issues and empirical findings. In E. L. Worthington (Ed.), Handbook of forgiveness. New York, NY: Brunner-Routledge.

Woodyatt, L. y Wenzel, M. (2014). A needs-based perspective on self-forgiveness: Addressing threat to moral identity as a means of encouraging interpersonal and intrapersonal restoration. Journal of Experimental Social Psychology, 50, 125-135.

Woodyatt, L., Wenzel, M. y Griffin, B. J. (Eds.). (2017). Handbook of the psychology of self-forgiveness. Springer: International Publishing.

Financiación: Este trabajo fue financiado por el proyecto: "El uso problemático de las redes sociales virtuales y las continuidades entre el bullying tradicional y el cyberbullying desde la perspectiva de género", subvencionado por el Fondo Europeo de Desarrollo Regional (FEDER) y por la Consejería de Economía, Conocimiento, Empresas y Universidad, de la Junta de Andalucía, en marco del programa operativo FEDER Andalucía 2014-2020 (ref. UP0-1265142). 